\title{
PREVISÃO DA PRODUÇÃO DE MILHO NO BRASIL POR MEIO DE MODELOS ESTATÍSTICOS LINEARES
}

\author{
MARTINELLO, Larissa Maria ${ }^{1}$ \\ RODRIGUES, Samuel Bellido ${ }^{2}$ \\ HICKMANN, Tásia ${ }^{3}$ \\ CORRÊEA, Jairo Marlon ${ }^{4}$ \\ THOMAZ, Diego Venâncio ${ }^{5}$ \\ RIBEIRO, Lucas da Silva ${ }^{6}$
}

\begin{abstract}
Recebido em: 2020.08.12
Aprovado em: 2021.04.01

ISSUE DOI: $10.3738 / 1982.2278 .3830$

RESUMO: O conhecimento de técnicas que permitam obter informações da tendência futura da produção é fundamental para o gestor rural. Diante disso, a finalidade desse trabalho foi realizar previsões. Para isso, foram utilizados modelos de séries temporais implementados no software livre R da produção brasileira de milho para as safras 2017/2018, 2018/2019 e 2019/2020. Foram aplicadas as metodologias ARIMA (Autoregressive Integrated Moving Average - Autorregressivo Integrado de Médias Móveis) e ETS (Error, Trend, Seasonal - Erro, Tendência, Sazonal). Ambos modelos provaram ser adequados. O modelo que apresentou os melhores resultados de previsão foi o ARIMA, cujo erro percentual médio absoluto das previsões foi menor que o apresentado no modelo ETS, quando comparado com os dados reservados para verificação da eficiência preditiva dos modelos ajustados. Os resultados demonstram a aplicabilidade dos modelos de previsão e ferramentas computacionais de fácil utilização. Tais técnicas visam contribuir no processo de tomada de decisão e planejamento por parte do gestor rural, que vê a cultura do milho, nos últimos anos, apresentar recordes de produção e ser um dos principais cultivares que contribui com a economia do Brasil.
\end{abstract}

Palavras-chave: Séries temporais. ARIMA. ETS.

\section{FORECASTING CORN PRODUCTION IN BRAZIL THROUGH LINEAR STATISTICAL MODELS}

SUMMARY: Knowledge of techniques to obtain information on the future production trend is essential for rural managers. Therefore, the purpose of this work was to make predictions. For that, time series models implemented in free software R of Brazilian corn production for the 2017/2018, 2018/2019 and 2019/2020 harvests were used. ARIMA (Autoregressive Integrated Moving Average) and ETS (Error, Trend, Seasonal - Error, Trend, Seasonal) methodologies were applied. Both models proved to be suitable. The model that presented the best forecast results was ARIMA, whose mean absolute percentage error of the predictions was less than that presented in the ETS model, when compared with the data reserved for verifying the predictive efficiency of the adjusted models. The results demonstrate the applicability of prediction models and computational tools that are easy to use. Such techniques aim to contribute to the decision-making and planning process by the rural manager, who has seen the corn crop in recent years, present production records and be one of the main cultivars that contributes to the Brazilian economy.

Keywords: Time series; ARIMA; ETS.

\section{INTRODUÇÃOO}

\footnotetext{
${ }^{1}$ https://orcid.org/0000-0001-7513-1388. Discente do curso de Engenharia de Produção na UTFPR (Medianeira-Pr).

2 https://orcid.org/0000-0002-9289-6670. Docente do Magistério Superior na UTFPR (Medianeira-Pr).

3 https://orcid.org/0000-0001-5147-700X . Docente do Magistério Superior na UTFPR (Medianeira-Pr).

4 https://orcid.org/0000-0002-7778-8102. Docente do Magistério Superior na UTFPR (Medianeira-Pr).

5 https://orcid.org/0000-0002-4945-3065. Docente do Magistério Superior na UTFPR (Medianeira-Pr).

${ }^{6}$ https://orcid.org/0000-0001-6169-6759. Docente do Magistério Superior na UTFPR (Medianeira-Pr).
} 
A produção agrícola vem se destacando cada vez mais na economia global. Em 2017, especificamente no Brasil, o agronegócio foi o protagonista da economia nacional. Com uma safra recorde de grãos que atingiu o patamar de 237,7 milhões de toneladas, o faturamento em dólares cresceu 12\% em relação ao ano anterior, fechando em 96 bilhões de dólares. Além disso, o setor avançou em 13\%, dando grande impulso ao PIB (Produto Interno Bruto) nacional (DINO, 2018).

Em 2019, o PIB do agronegócio brasileiro cresceu 3,81\%, revelando um desempenho significativamente superior à média da economia brasileira. Com isso, nesse mesmo ano, o agronegócio ampliou sua participação na economia, passando a representar $21,4 \%$, frente a $21,1 \%$ do ano anterior (CNA; CEPEA, 2020).

A produção brasileira de milho tem apresentado tendência de elevação desde o fím da década de 80. Vale aqui mencionar os maiores produtores mundiais desse grão: Estados Unidos, China, Brasil e Argentina, que juntos representam $70 \%$ da produção mundial. Atualmente, além de ser o terceiro maior produtor, o Brasil ainda se destaca como o segundo maior exportador mundial de milho (CONAB, 2019).

A importância econômica da produção de milho é caracterizada pelas diversas formas de sua utilização, que vai desde a alimentação animal até a indústria de alta tecnologia fazendo do milho um dos mais importantes produtos do setor agrícola brasileiro. O milho é cultivado em praticamente todo o território nacional. As regiões sul, sudeste e centro-oeste concentram $77 \%$ da área plantada e 92\% da produção brasileira (DUARTE; MATTOSO; GARCIA, 2017).

Diante da sua elevada importância para a economia nacional, a Companhia Nacional de Abastecimento (CONAB) realiza previsões anuais para as safras de milho em todas as regiões do país, as quais são baseadas nas safras anteriores, fornecendo informações e conhecimentos relevantes aos agentes envolvidos nos desafios da agricultura, da segurança alimentar e nutricionais e do abastecimento do país (CONAB, 2019).

As projeções procuram indicar direções do desenvolvimento e fornecer subsídios aos formuladores de políticas públicas quanto às tendências dos principais produtos do agronegócio. As tendências indicadas permitem identificar trajetórias possíveis, bem como estruturar visões de futuro do agronegócio no contexto nacional e mundial para que o país continue crescendo e conquistando novos mercados (MAPA, 2019).

Segundo Ionics (2019), pode-se afirmar que a tecnologia de inovação será um fator cada vez mais determinante para o sucesso dos empreendimentos rurais, de modo que é possível focar em uma gestão profissional voltada a aumentar a produtividade e a reduzir custos. Ter conhecimento da tendência no agronegócio é muito relevante para o gestor rural, uma vez que o auxilia na elaboração de panoramas e tomadas de decisões mais assertivas para os negócios 
relacionados ao seu empreendimento, sobretudo em um contexto de transformação contínua, que é a realidade vivida pelo setor.

Vista à importância do agronegócio na economia do país e o interesse por conhecer possíveis cenários futuros da produtividade de diversas culturas, em especial neste trabalho a do milho, justifica-se o estudo e a aplicação de técnicas estatísticas nas séries históricas da produção brasileira no agronegócio. É importante mencionar que as técnicas de análise e previsão de séries temporais podem ser aplicadas ao banco de dados de produção de milho, soja, algodão, cana-deaçúcar, suínos, frangos, bovinos, entre outros, fornecendo um horizonte de produção (RESENDE, 2016).

O presente trabalho tem como objetivo realizar a previsão da produção de milho no Brasil para as safras de 2017/2018, 2018/2019 e 2019/2020 por meio dos modelos estatísticos autorregressivo integrado médias móveis (ARIMA) e modelo de alisamento exponencial via espaço de estados (ETS), utilizando o software livre R.

\section{FUNDAMENTAÇÃO TEÓRICA}

\section{Séries Temporais}

Uma série temporal é um conjunto de dados numéricos sequencial no tempo capaz de produzir uma descrição do passado, e a partir de um procedimento lógico e com o uso desses dados históricos, realizar previsões. Se os dados passados são indicativos do que se esperar no futuro, pode-se então postular um modelo matemático que é representativo ao processo que gerou os valores da série (NOGUEIRA, 2009).

Para Mastrangelo (2013), os principais objetivos ao se estudar séries temporais são: compreensão, modelagem, predição dos valores futuros e controle de algum sistema para o qual a série temporal é uma medida de desempenho.

Segundo Chiba e Luna (2020) o uso de modelos de séries temporais é de suma importância no âmbito econômico atual, além de que há grande disponibilidade de softwares de baixo custo, ou livres, que podem ser usados para realizar previsões. Na literatura é muito empregada a métrica de erro MAPE, para mensurar o ajuste dos modelos aos dados. Esta métrica representa uma quantidade adimensional e avalia a precisão do modelo de previsão, é possível observar com facilidade a eficiência dos métodos, pois seus resultados são expressos em porcentagem (AASIM; SINGH; MOHAPATRA, 2019). 


\section{ARIMA}

Os modelos ARIMA são modelos estatísticos lineares que realizam previsões de séries temporais. Os termos autorregressivos correspondem às defasagens da série transformada (série obtida após realizar o processo de diferenciação que a torna estacionária na média) e as médias móveis às defasagens dos erros aleatórios. Segundo Büyükşahin e Ertekin (2019), modelos lineares como ARIMA fornecem melhor precisão de previsão com dados de séries temporais estacionários. O termo "integrado" refere-se ao processo de diferenciação da série original para torná-la estacionária (MORETTIN; TOLOI, 2018).

O modelo tem como premissa básica que a série temporal é gerada por um processo estocástico, cuja natureza pode ser representada através de um modelo matemático. A notação empregada para designá-lo é usualmente $\operatorname{ARIMA~(p,~d,~q),~onde~"~} p$ " representa o número de parâmetros autorregressivos, "d" o número de diferenciações para que a série se torne estacionaria e "q" o número de parâmetros de médias móveis (BOX; JENKINS; REINSEL; LJUNG, 2015).

O modelo ARIMA (p, d, q) pode ser escrito como:

$$
\emptyset(B)(1-B)^{d} Z_{t}=\theta(B) \varepsilon_{t}
$$

onde $\varepsilon_{t}$ é ruído branco, $\emptyset(B)$ e $\theta(B)$ são o polinômio autorregressivo e o polinômio de médias móveis, respectivamente, $(1-B)$ representa o operador diferença e $d$ representa o número de diferenças.

\section{Suavização Exponencial}

Os métodos de suavização exponencial com representação em espaço-estado, ou simplesmente ETS, produzem previsões pontuais bem como intervalos de previsão. As previsões são geradas por meio da média ponderada das observações anteriores, com pesos decaindo de forma exponencial, ou seja, os maiores pesos são associados às observações mais recentes (HYNDMAN et al. 2008).

Na modelagem ETS tem-se o erro (E), a tendência $(T)$ e a sazonalidade $(S)$, onde o erro pode ser aditivo (A) ou multiplicativo (M); a tendência pode não ocorrer (N), ser aditiva (A), aditiva amortecida (Aa), multiplicativa (M) ou multiplicativa amortecida (Ma); e a sazonalidade pode não ocorrer $(\mathrm{N})$, ser aditiva $(\mathrm{A})$ ou multiplicativa $(\mathrm{M})$. As 30 equações do modelo ETS com as possibilidades descritas acima podem ser encontrados em Hyndman, (2008) pág. 21 e 22.

\section{Software $\mathbf{R}$}

O R é um software computacional e gráfico que possui duas grandes vantagens que se faz jus mencionar: a primeira é que se trata de um software de código livre, onde os usuários podem 
estudar seu funcionamento e adaptá-lo às suas necessidades; a segunda é que o mesmo pode ser utilizado para realizar qualquer modelagem computacional, desde que compatível com suas capacidades, a partir da criação de funções próprias, uso de pacotes, desenvolvimento de códigos para a resolução de problemas numéricos relacionados a diversas aplicações (RITTER; THEY; KONZEN, 2019).

De um modo geral, o R é uma ferramenta de grande valor para armazenar e manipular dados, realizar cálculos, testes estatísticos e análises exploratórias e produzir gráficos (RITTER; THEY; KONZEN, 2019).

\section{Pacote forecast}

O pacote forecast é constituído de métodos e ferramentas que permitem obter previsões automáticas de séries temporais (HYDMANN, et. al., 2019).

Dentre as várias funções contidas no pacote forecast, as utilizadas neste trabalho foram as funções de seleção automática auto.arima( ) e ets( ). Tais funções priorizam a escolha de um modelo parcimonioso, ou seja, um modelo que explique bem o comportamento dos dados de uma série com um número mínimo de parâmetros (HYNDMAN et al., 2019).

A escolha automática do modelo ARIMA utilizando a função auto.arima( ) se dá por meio do teste da raiz unitária e o critério de informação AICc (Hyndman \& Athanasopoulos, 2008), enquanto que a escolha do modelo automático ETS utilizando a função ets( ) considera todos os 30 modelos e seleciona aquele com o menor critério de informação, que por default é AICc (HYNDMAN; KHANDAKAR, 2008; HYNDMAN et al., 2019).

\section{MATERIAL E MÉTODO}

Para aplicação dos modelos ARIMA e ETS foram utilizados dados da produção brasileira de milho no período de 1977 a 2020, que compreendem as safras 1977/1978 a 2019/2020, obtidos por meio do site da $\mathrm{CONAB}^{7}$ e que estão representados no Gráfico 1. Os dados das safras 2017/2018 a 2019/2020 foram utilizadas apenas para comparar com as previsões obtidas por meio dos modelos estatísticos e não foram utilizados na modelagem. Convém citar que os valores da produção da safra de 2019/2020 são dados de previsão feita pela própria CONAB e que são atualizadas mensalmente, e o valor utilizado é o que foi obtido no dia 10 de julho de 2020.

\footnotetext{
${ }^{7}$ https://www.conab.gov.br/info-agro/safras/graos (Acesso em: 10 de julho de 2020)
} 
Gráfico 1. Produção anual de milho entre 1977 e 2020

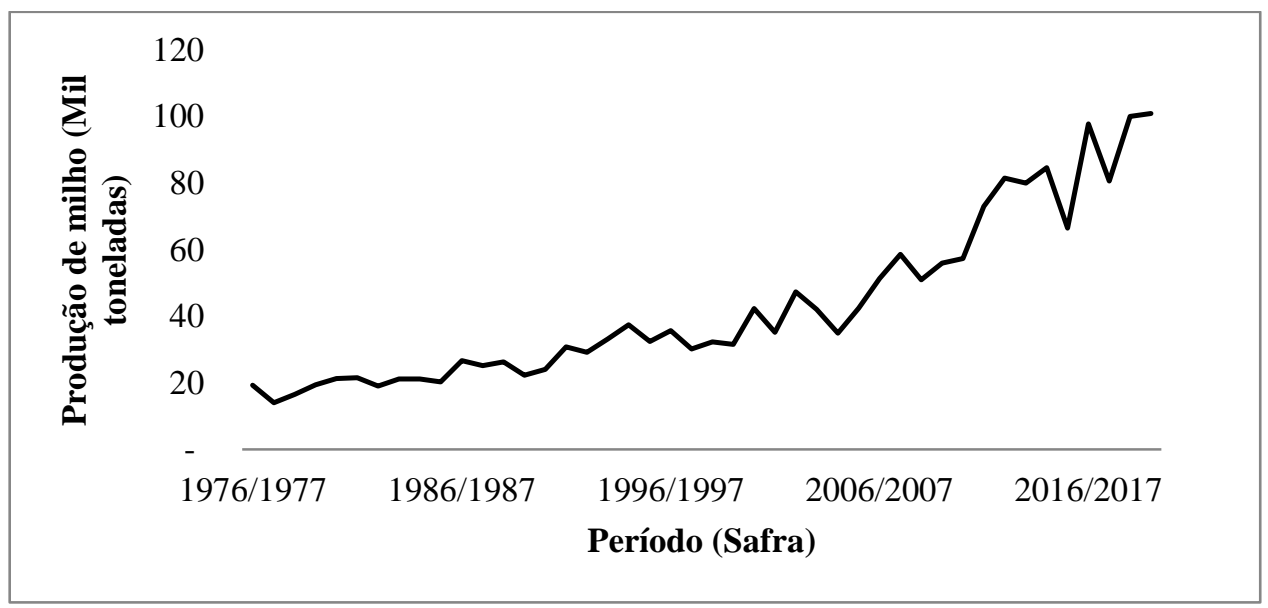

Fonte: Elaborado pelos Autores (2020)

Ao analisar os dados da produção do milho, verificou-se que a produção saltou de 20 milhões de toneladas na safra de 1977/1978 para aproximadamente 98 milhões de toneladas na safra 2016/2017, crescimento evidenciado pelo aumento da produtividade, com a introdução de novas técnicas de manejo, novos fertilizantes e aumento da área cultivada em todo país.

Para aplicação dos modelos e organização dos dados utilizou-se o pacote forecast do programa R Studio, que utiliza critérios de informação privilegiando modelos parcimoniosos, os quais envolvem um número mínimo de parâmetros possíveis a serem estimados e que explique bem o comportamento dos dados da série. Posteriormente, foram calculadas as principais métricas de erros: Erro Percentual Médio Absoluto (MAPE), Erro Médio Absoluto (MAE) e Raiz do Erro Quadrático Médio (RMSE) a fim de se evidenciar a eficácia dos modelos junto aos dados. A Figura 1 descreve as etapas desenvolvidas para obtenção dos resultados.

Figura 1. Fluxograma da metodologia aplicada

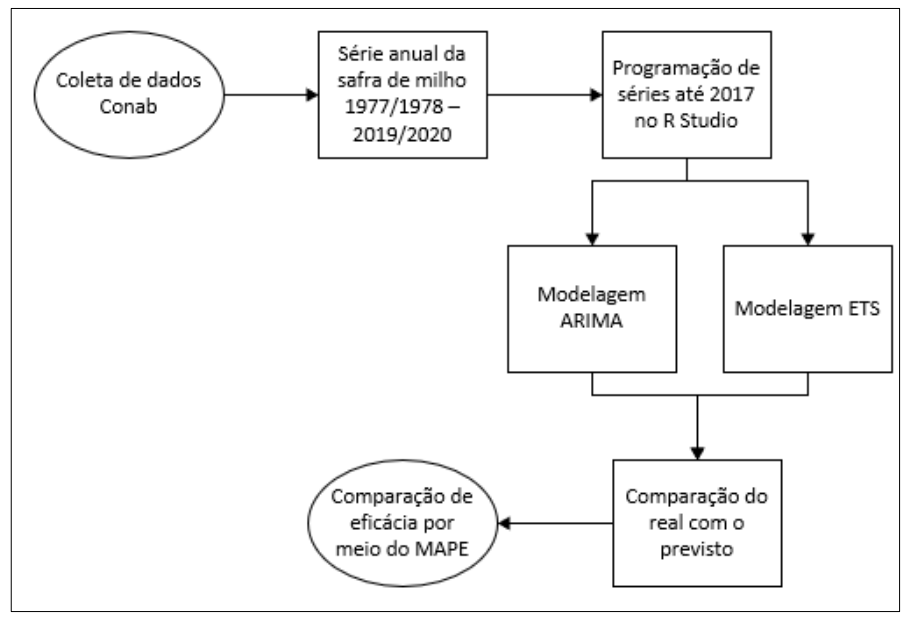

Fonte: Elaborado pelos Autores (2020) 


\section{RESULTADO E DISCUSSÃO}

Foram utilizados os métodos ARIMA e ETS e a metodologia descrita na seção anterior, aplicou-se o pacote forecast aos dados a partir do software $\mathrm{R}$, e assim foram gerados os parâmetros dos modelos que melhores se ajustaram. Aplicando-se a modelagem ARIMA na série apresentada, obteve-se o modelo $\operatorname{ARIMA}(1,1,0)$, os parâmetros obtidos podem ser vistos na Tabela 1:

Tabela 1. Parâmetros de saída modelagem ARIMA

\begin{tabular}{cc}
\hline ARIMA $(1,1,0)$ \\
\hline \multicolumn{3}{c}{ Coeficientes: } \\
ar1 & drift \\
$-0,5837$ & 1756,8808 \\
s.e. 0,1685 & 690,2087 \\
\hline
\end{tabular}

Fonte: Elaborado pelos Autores (2020)

Realizaram-se os testes de Ljung-Box, o qual se constitui em uma ferramenta diagnóstica utilizada para testar a falha do ajuste de um modelo de série temporal e é aplicado aos seus resíduos após o ajustamento de um modelo (SOUZA, 2016). Este teste apresentou P-valor = 0,03734 e o teste de normalidade de Jarque - Bera, com P-valor $=0,08$, descrito respectivamente em Ljung e Box (1978) e Jarque e Bera (1987), indicando que as suposições de independência e a normalidade dos resíduos são atendidas ao nível de significância de 1\%, elementos essenciais para aceitação do modelo.

O Gráfico 2 apresenta a relação entre os valores reais e os valores obtidos pelo modelo ARIMA, no período de 1977-2017.

Gráfico 2. Série original e prevista da modelagem ARIMA

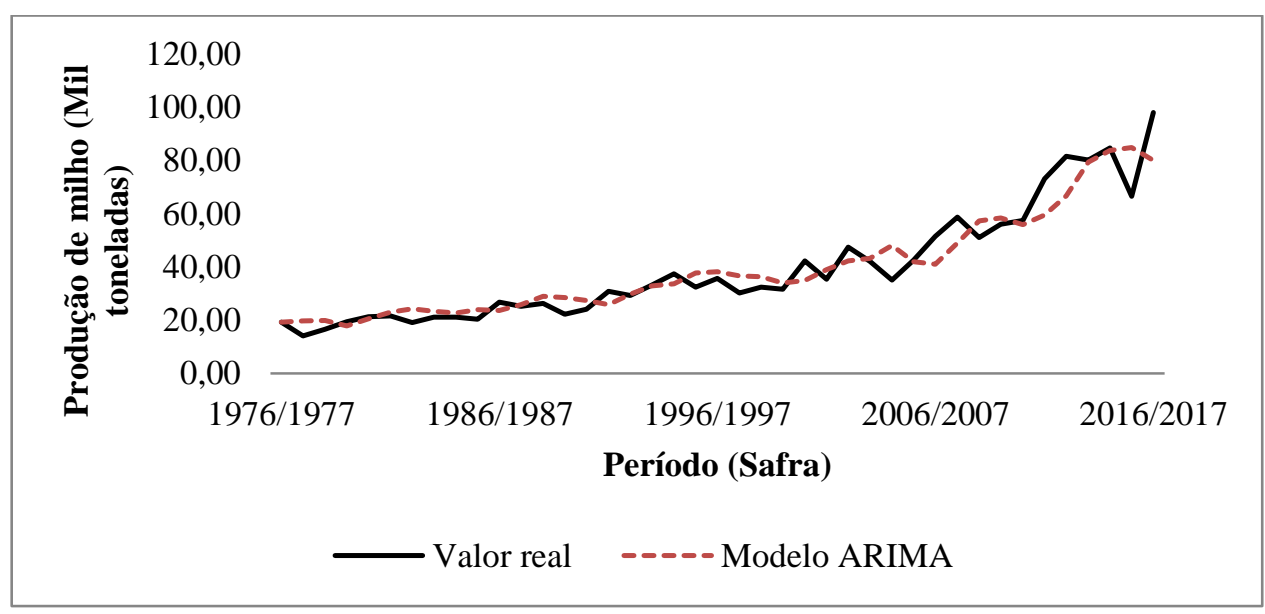

Fonte: Elaborado pelos Autores (2020) 
A análise residual, Figura 2, apresenta os resíduos indicando aleatoriedade, a função autocorrelação e os resíduos comparados com a distribuição normal.

Figura 2. Análise residual da modelagem ARIMA

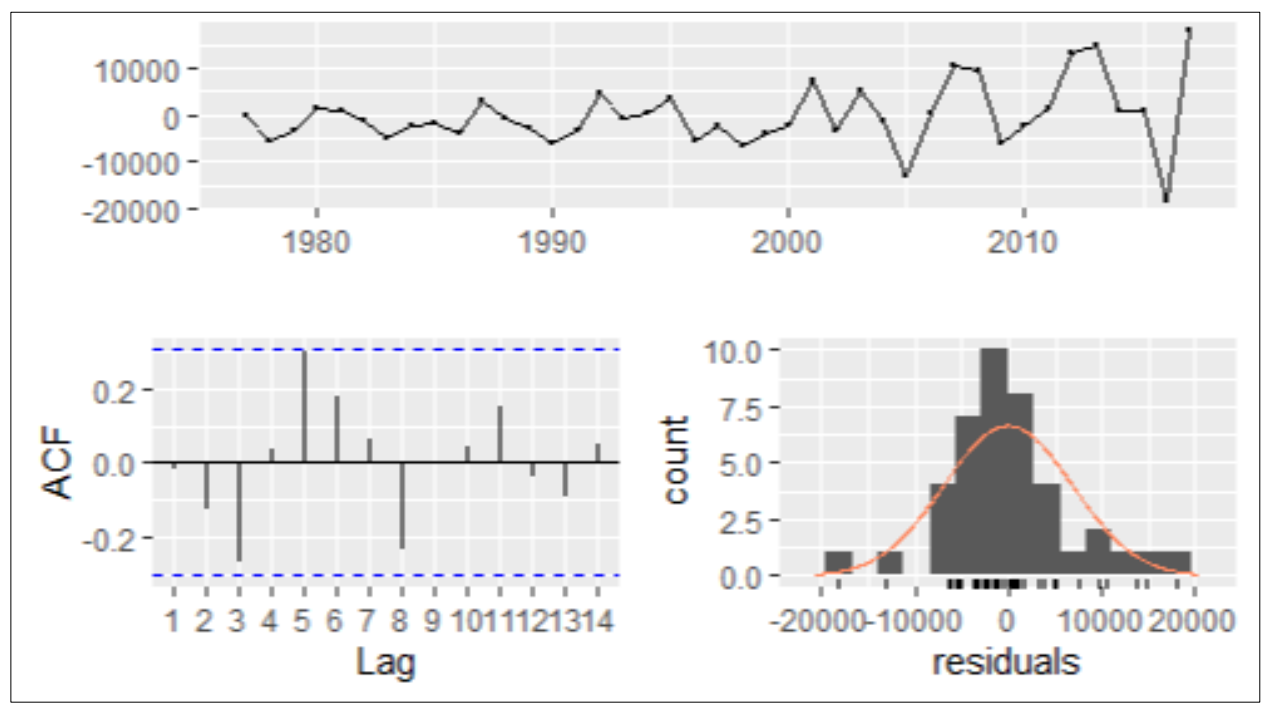

Fonte: Elaborado pelos Autores (2020)

Após a validação do modelo ARIMA(1,1,0), as previsões para as safras de 2017/2018, 2018/2019 e 2019/2020 ${ }^{8}$ foram obtidas, as quais podem ser observadas no Gráfico 3, além da previsão, apresenta os intervalos de confiança de 95\%, superior e inferior, e o valor real da série.

Gráfico 3. Previsão ARIMA

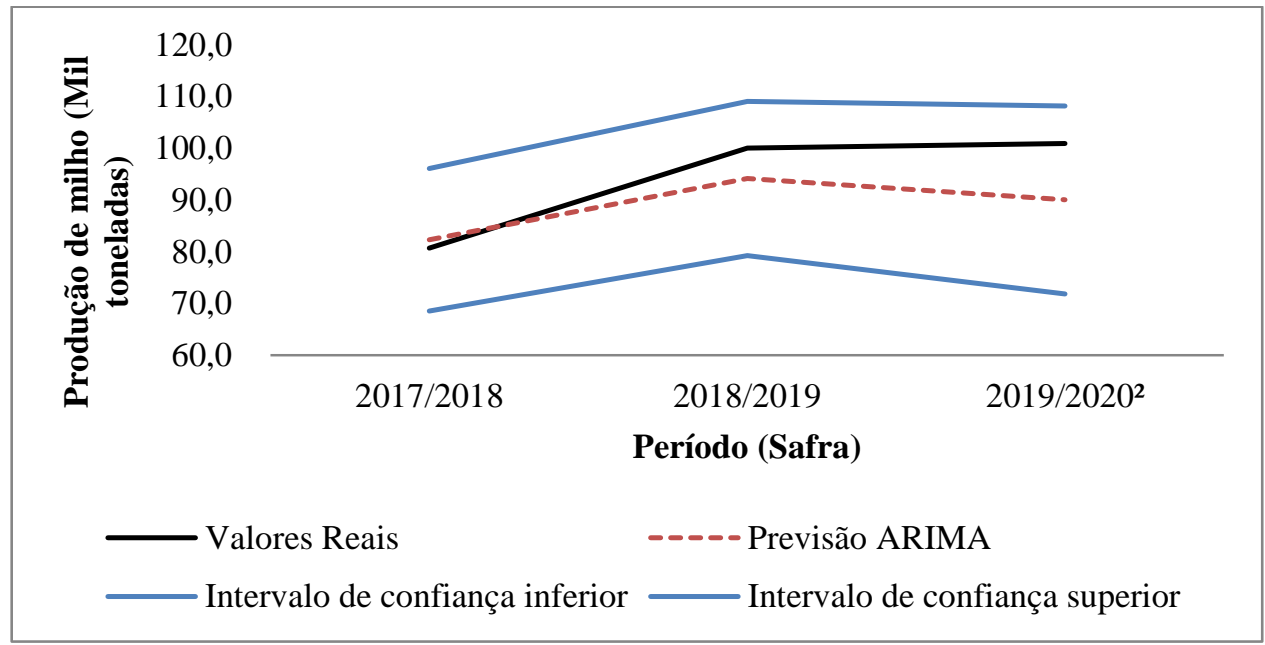

Fonte: Elaborado pelos Autores (2020)

Na sequência foi realizada a modelagem ETS na série de produção do milho, a qual, segundo Costa (2017), analisa os dados de demanda no tempo e define qual o melhor modelo de

${ }^{2}$ Previsão feita pelo CONAB atualizada até a data da coleta dos dados.

Nucleus, v.18, n.1, abr. 2021 
previsão a ser utilizado, de forma a prevalecer aquele que fornece uma previsão com o menor erro possível. Esta modelagem retornou o modelo expresso por ETS(M, A, N), ou seja, possui erros multiplicativos, tendência aditiva e não apresenta sazonalidade, também denominado método linear de Holt com erros multiplicativos. Os parâmetros do modelo podem ser vistos na Tabela 2.

Tabela 2. Parâmetros de saída modelagem ETS

\begin{tabular}{c}
\hline ETS $(\mathrm{M}, \mathrm{A}, \mathrm{N})$ \\
\hline Parâmetros de suavização: \\
Alpha $=0,1846 \quad$ Beta $=0,0816$ \\
Estados iniciais: \\
$1=16655,5002 \quad \mathrm{~b}=647,5613$ \\
\hline Fonte: Elaborado pelos Autores $(2020)$
\end{tabular}

O Gráfico 4 mostra a relação entre o valor real e o previsto pelo modelo ETS(M, A, N) no período de 1977-2017. É notória a similaridade entre as duas curvas, o que indica uma boa aderência do modelo.

Gráfico 4 - Série original e prevista da modelagem ETS

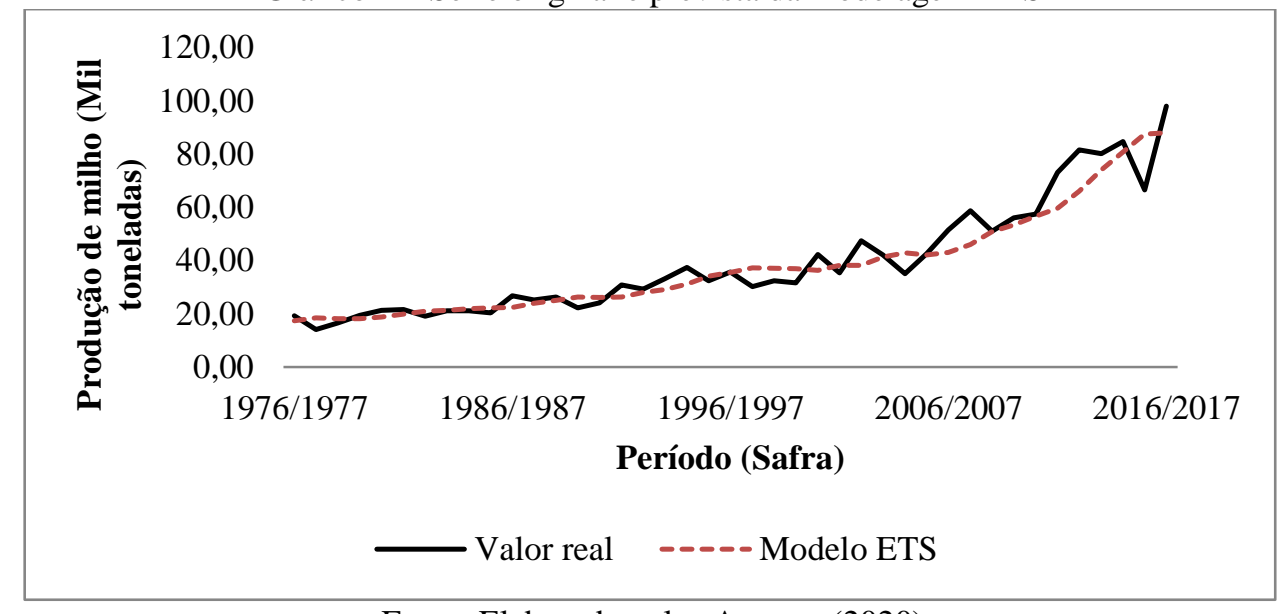

Fonte: Elaborado pelos Autores (2020)

Além disso, a previsão pelo modelo ETS(M, A, N) para as safras de 2017/2018, 2018/2019 e 2019/20203, assim como o valor real e os intervalos de confiança de $95 \%$, superior e inferior, encontram-se no Gráfico 5. 
Gráfico 5. Previsão ETS

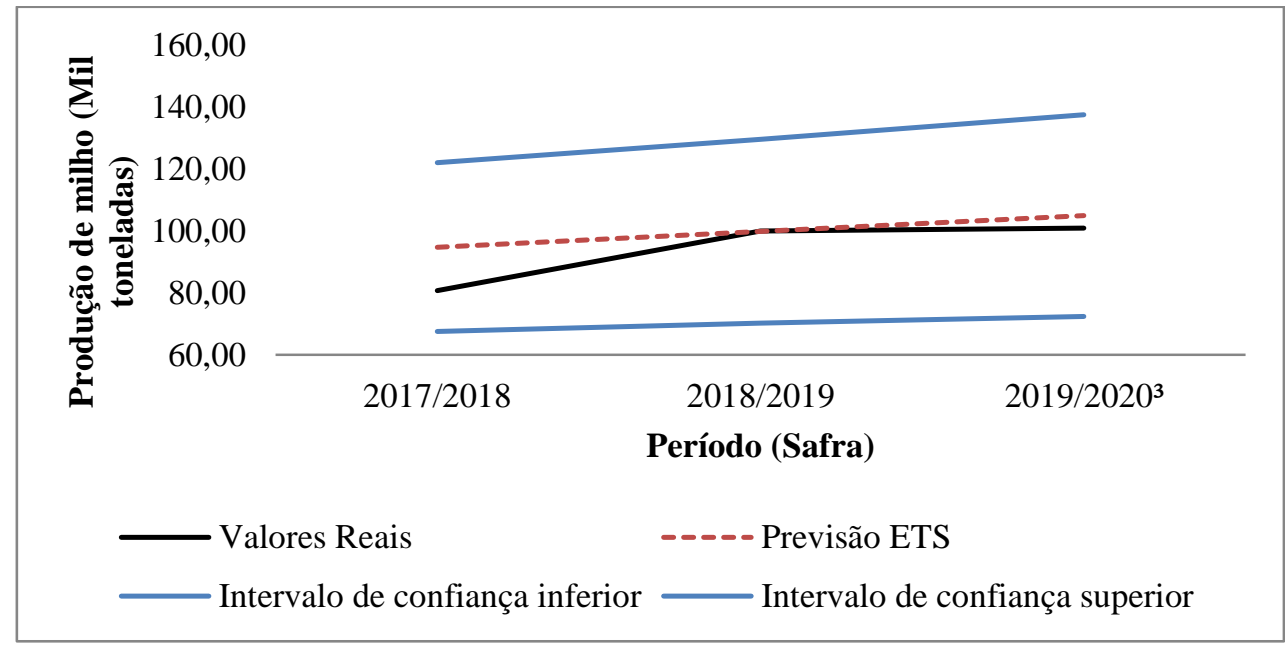

Fonte: Elaborado pelos Autores (2020)

A análise gráfica das previsões obtidas pelo modelo $\operatorname{ARIMA}(1,1,0)$ mostram que os valores seguem o comportamento dos dados reais, ainda que as previsões para as safras 2018/2019 e 2019/2020 , ficaram abaixo do valor real, refletindo que a tendência de aumento da produção pode não ser a mesma dos anos anteriores.

A mesma análise pode ser estendida para as previsões obtidas pelo modelo ETS (M, A, $\mathrm{N}$ ), porém nota-se nitidamente que as previsões refletem uma tendência de crescimento para as safras no período analisado, tal tendência foi capturada no momento da modelagem, e assim concluir que, mantidas as condições, o modelo prevê tendência de aumento na produção. $\mathrm{O}$ valor para a safra de 2018/2019 ficou abaixo do real enquanto para os demais valores acima.

A Tabela 3 apresenta os valores reais, os valores previstos obtidos a partir dos modelos selecionados e seus respectivos erros MAE (erro absoluto médio), RMSE (raiz do erro médio quadrático) e MAPE (erro percentual absoluto médio), para as safras 2017/2018, 2018/2019 e $2019 / 2020^{10}$.

Tabela 3. Previsões de produção de milho e erros

\begin{tabular}{c|c|c|c}
\hline Período (Safra) & Real (Mil toneladas) & ARIMA (1, 1, 0) & ETS (M, A, N) \\
\hline $2017 / 2018$ & 80709,5 & 82347,55 & 94798,12 \\
$2018 / 2019$ & 100042,7 & 94174,9 & 99883,88 \\
$2019 / 2020^{4}$ & 100992,9 & 90053,41 & 104969,6 \\
\hline \multicolumn{2}{c|}{$\begin{array}{c}\text { MAE } \\
\text { RMSE }\end{array}$} & 6148,445 & 6074,727 \\
\multicolumn{2}{c|}{ MAPE (\%) } & 7229,259 & 8452,394 \\
\end{tabular}

Fonte: Elaborado pelos Autores (2020)

\footnotetext{
${ }^{3}$ Previsão feita pelo CONAB atualizada até a data da coleta dos dados.

${ }^{4}$ Previsão feita pelo CONAB atualizada até a data da coleta dos dados.

Nucleus, v.18, n.1, abr. 2021
} 
Diante dos resultados e da aceitação de ambos os modelos, mas a partir de uma possível escolha, destaca-se que o modelo $\operatorname{ARIMA}(1,1,0)$ obteve melhores resultados nas previsões das safras 2017/2018, 2018/2019 e 2019/2020, pois o MAPE obtido pelo modelo $\operatorname{ARIMA}(1,1,0)$ foi de $6,2 \%$ e RMSE de 7229,259, frente ao MAPE de 7,18\% e RMSE 8452,394 do modelo ETS(M, A, N).

\section{CONCLUSÃO}

O presente trabalho apresenta previsões da produção de milho no Brasil para as safras 2017/2018, 2018/2019 e 2019/2020, a partir de modelos ARIMA e ETS. Ambos os modelos se adaptam bem aos dados sendo considerados modelos aceitos.

Com os resultados obtidos na previsão das safras de 2017/2018, 2018/2019 e 2019/2020 e estes comparados com os dados reais nos faz concluir que o modelo $\operatorname{ARIMA}(1,1,0)$ apresenta melhor resultado, quando comparado o erro percentual médio frente ao modelo ETS(M, A, N).

Tais resultados ressaltam a importância da investigação de ferramentas que visam contribuir com o planejamento do agronegócio brasileiro. A análise de séries temporais com a adoção de softwares livre é uma dessas ferramentas, e sua utilização na previsão do cultivar de milho é de interesse nacional já que a comercialização de grãos tem grande impacto no PIB nacional.

Além disso, a utilização de softwares livres e pacotes de previsão automáticos reduzem custos, são de simples utilização e atendem às propriedades matemáticas impostas pelos modelos preditivos.

Sendo assim, como sugestão para futuras pesquisas, recomenda-se a investigação de outros modelos como, por exemplo, as redes neurais artificias (RNA) e modelos que considerem a inclusão de variáveis exógenas como por exemplo o modelo ARIMAX, bem como a combinação de previsões. Outro destaque será a investigação do software livre PYTHON e ferramentas que possam ser disponibilizas em dispositivos portáteis a serem disponibilizados de maneira gratuita aos produtores.

\section{REFERÊNCIAS}

AASIM; SINGH, S.N.; MOHAPATRA, Abheejeet. Repeated wavelet transform based ARIMA model for very short-term wind speed forecasting. Renewable Energy, [S.L.], v. 136, p. 758768, jun. 2019. Elsevier BV. http://dx.doi.org/10.1016/j.renene.2019.01.031.

BOX, G. E. P.; JENKINS, G. M.; REINSEL, G. C.; LJUNG, G. M. Time series analysis: Forecasting and control (5th ed). Hoboken, New Jersey: John Wiley \& Sons, 2015. 
BÜYÜKşAHIN, Ü. Ç.; ERTEKIN, Ş. Improving forecasting accuracy of time series data using a new ARIMA-ANN hybrid method and empirical mode decomposition. Neurocomputing, [S.L.], v. 361, p. 151-163, out. 2019. Elsevier BV. http://dx.doi.org/10.1016/j.neucom.2019.05.099.

CHIBA, G. I.; LUNA, M. M. M. Time series models: an analysis of demand forecast accuracy for a product line in a clothing company. Revista Gestão da Produção Operações e Sistemas, [S.L.], v. 15, n. 4, p. 219-251, 24 nov. 2020. A Fundação para o Desenvolvimento de Bauru (FunDeB). http://dx.doi.org/10.15675/gepros.v15i4.2664.

CNA, Confederação da Agricultura e Pecuária do Brasil; CEPEA, Centro de Estudos Avançados em Economia Aplicada. PIB do agronegócio cresce 3,81\% em 2019. Disponível em: $<$ https://www.cnabrasil.org.br/boletins/pib-do-agronegocio-cresce-3-81-em2019\#: :text=O\%20PIB\%20(Renda)\%20do\%20agroneg\%C3\%B3cio,21\%2C1\%25\%20em\%202 018.> Acesso em 28 de julho de 2020.

CONAB - Companhia Nacional de Abastecimento. Perspectiva para a agropecuária. v.7 Brasília, 2019.

COSTA, C. S. Controle de estoques em uma empresa do setor alimentício pela aplicação do lote econômico de produção. Niterói, RJ, 2017.

DINO, O. Setor agrícola é o impulsionador da economia nacional. Revista Exame. Disponível em: $<$ https://exame.com/negocios/dino_old/setor-agricola-e-o-impulsionador-da-economianacional/> Acesso em 16 de julho de 2020.

DUARTE, J. A.; MATTOSO, M. J.; GARCIA, J. C. Importância socioeconômica do milho. Disponível em:

<https://www.agencia.cnptia.embrapa.br/gestor/milho/arvore/CONTAG01_8_168200511157.htm l\#> Acesso em: 27 de julho de 2020.

JARQUE, C. M.; BERA, A. K. A test for normality of observations and regression residuals. Int. Stat. Rev., Woodbury, v.55, p.163-172, 1987.

HYNDMAN, R. J., ATHANASOPOULOS, G. Forecasting: principles and practice. OTexts, 2018.

HYNDMAN, R. J.; ATHANASOPOULOS, G.; BERGMEIR, C.; CACERES, G; CHHAY, L.; O'HARA-WILD, M.; PETROPOULOS, F.; RAZBASH, S.; WANG, E.; YASMEEN, F.

Forecast: Forecasting functions for time series and linear models. 2019. R package version 8.5 .

HYNDMAN, R.J., KHANDAKAR, Y. Automatic Time Series Forecasting: The forecast Package for R. Journal of Statistical Software, 2008.

HYNDMAN, R.J., KOEHLER, A. B., ORD, J. K.; SNYDER, R. D. Forecasting with Exponential Smoothing: The State Space Approach. Berlim: Springer-Verlag, 2008.

IONICS. Tendência para o agronegócio em 2020: expectativas para o setor no próximo ano. Disponível em: $<$ https://ionics.com.br/tendencia-para-o-agronegocio-em-2020-expectativas-parao-setor-no-proximo-ano/> Acesso em 28 de julho de 2020.

LJUNG, G. M; BOX, G. E. P. On a measure of a lack of fit in time series models. Biometrika, v. 65 , p. $297-303,1978$. 
MAPA, Ministério da Agricultura, Pecuário e Abastecimento. Projeções do agronegócio. Disponível em: $<$ https://www.gov.br/agricultura/pt-br/assuntos/politica-agricola/todaspublicacoes-de-politica-agricola/projecoes-do-agronegocio/projecoes-do-agronegocio-20182019-2028-2029> Acesso em 27 de julho de 2020.

MASTRANGELO C.M., SIMPSON J.R., MONTGOMERY D.C. Time Series Analysis. In: Gass S.I., Fu M.C. (eds) Encyclopedia of Operations Research and Management Science. Springer, Boston, 2013.

MORETTIN, P. A.; TOILOI, C. M. C. Análise de séries temporais: modelos lineares univariados. v. 1. Blucher, 2018.

NOGUEIRA, F. Modelagem e Simulação - Modelos de Previsão, 2009. Disponível em:< http://www.ufjf.br/epd042/files/2009/02/previsao1.pdf >Acesso em: 10 de julho de 2020.

RESENDE, L. F. S. Modelagem via séries temporais da Produção de Milho no Brasil, 2016. Disponível em:< http://repositorio.ufu.br/bitstream/123456789/17901/1/ModelagemSeriesTemporais.pdf> Acesso em 10 de julho de 2020.

RITTER, M. N.; THEY, N. H,; KONZEN, E. Introdução ao software estatístico R. Disponível em: $<$ http://professor.ufrgs.br/sites/default/files/matiasritter/files/apostila_introducao_ao_r__ritter_they_and_konzen.pdf $>$ Acesso em 10 de julho de 2020.

SOUZA, Francisca Mendonça. Modelos de previsão: aplicações à energia elétrica - ARIMA ARCH - AI e ACP. Curitiba: Appris, 2016. 